\title{
Effectiveness of Sodium-Glucose Cotransporter-2 Inhibitor as an Add-on Drug to GLP-1 Receptor Agonists for Glycemic Control of a Patient with Prader-Willi Syndrome: A Case Report
}

\author{
Yukio Horikawa - Mayumi Enya - Makie Komagata - Ken-ichi Hashimoto • \\ Masayo Kagami · Maki Fukami · Jun Takeda
}

Received: December 5, 2017 / Published online: January 15, 2018

(C) The Author(s) 2018. This article is an open access publication

\begin{abstract}
Introduction: Diabetes patients with Prader-Willi syndrome (PWS) are obese because of hyperphagia; weight control by dietary modification and medicine is required for glycemic control. There are several recent reports showing the effectiveness of GLP-1 receptor agonists (GLP-1RAs) for diabetes treatment in PWS.

Case Report: A 36-year-old Japanese male patient was diagnosed with PWS at 10 years of age. At age 16 years, he was diagnosed with
\end{abstract}

Enhanced content To view enhanced content for this article go to http://www.medengine.com/Redeem/ B02DF06055B051E2.

Electronic supplementary material The online version of this article (https://doi.org/10.1007/s13300018-0369-5) contains supplementary material, which is available to authorized users.

Y. Horikawa $(\bowtie) \cdot$ M. Enya $\cdot$ M. Komagata .

K. Hashimoto · J. Takeda

Department of Diabetes and Endocrinology, Gifu University Graduate School of Medicine, 1-1

Yanagido, Gifu 501-1194, Japan

e-mail: yhorikaw@gifu-u.ac.jp

Y. Horikawa · M. Enya $\cdot$ K. Hashimoto $\cdot$ J. Takeda Division of Clinical Genetics, Gifu University Hospital, 1-1 Yanagido, Gifu 501-1194, Japan

M. Kagami · M. Fukami

Department of Molecular Endocrinology, National Research Institute for Child Health and

Development, 2-10-1 Okura, Setagaya, Tokyo 157-

8535, Japan diabetes and began to take several kinds of oral hypoglycemic agents. At age 29 years, his BMI was $39.1 \mathrm{~kg} / \mathrm{m}^{2}$ and he was referred to our department for diabetes and obesity treatment. In the present case, the HbA1c was not improved by GLP-1RAs despite a 28-kg BW reduction, which included a $9-\mathrm{kg}$ loss of muscle. Apprehensive of further loss of muscle mass, basal insulin of insulin glargine was administered in addition to GLP-1RAs. Immediately after the addition of tofogliflozin, a sodiumglucose cotransporter-2 (SGLT2) inhibitor, the patient's HbA1c decreased dramatically with only about an additional 3\% BW reduction. We note an improvement in our case of lipid deposition in the pancreas confirmed by abdominal CT after the improvement of HbA1c. It is unknown whether this improvement of fatty pancreas was a cause or an effect of the improved glycemic control in the present case. Conclusion: This finding clearly supports the effectiveness of combining SGLT2 inhibitors with GLP-1RAs for treatment of patients with PWS and non-alcoholic fatty pancreas disease.

Keywords: Prader-Willi syndrome (PWS); Sodium-glucose cotransporter-2 (SGLT2) inhibitor; GLP-1 receptor agonists (GLP-1RAs); Non-alcoholic fatty pancreas disease (NAFPD) 


\section{INTRODUCTION}

Prader-Willi syndrome (PWS) is a complex multisystem genetic disorder with an incidence of one in 10,000-15,000 live births in Japan. PWS is an imprinting disorder that arises from lack of expression of paternally inherited genes on chromosome 15q11-q13. The syndrome has a characteristic phenotype that includes neonatal hypotonia, hyperphagia, hypogonadism, obesity, short stature, and cognitive and behavioral disabilities. In adult PWS, the most difficult to treat feature is morbid obesity due to hyperphagia caused by an absence of satiety and decreased energy expenditure. Severe obesity increases risk of developing diabetes mellitus [1-3]. While diet therapy is fundamentally important in the treatment of diabetes, it is problematic in patients with PWS, and requires use of insulin and other injected drugs as well as oral medications. During the last decade, many new medicines for treating diabetes have become available, but there are as yet no guidelines on these drugs for adult patients with PWS. This is the first report of the effectiveness of a sodium-glucose cotransporter2 (SGLT2) inhibitor as an add-on drug to GLP-1 receptor agonists (GLP-1RAs) for glycemic control of adult patients with PWS.

\section{CASE REPORT}

A 36-year-old Japanese male patient was born at 36 weeks' gestation via vaginal breech delivery. He had newborn asphyxia and an Apgar score of 4. Because of hypotonia, his height could not be measured and his weight was $2150 \mathrm{~g}$. At the age of 10 years he was diagnosed with PWS on the basis of typical clinical findings including being a floppy infant and having almond shaped eyes, scoliosis, obesity (weight more than $20 \%$ of standard), mental retardation (IQ of 61), and hypogonadism with bilateral cryptorchidism. At age 12 years, he was referred to a pediatric endocrinologist for assessment of his pituitary function and glucose tolerance. Oral glucose and insulin tolerance test showed impaired glucose tolerance (IGT) and little reaction of growth hormone. GH therapy was not done because of the IGT level and severe obesity. At age 16 years, he was diagnosed with diabetes and began to take several kinds of oral hypoglycemic agents.

At age 28 years, he was referred to our department of diabetes and endocrinology for diabetes and obesity treatment. At that time, he was obese with body weight of $90.6 \mathrm{~kg}$, height of $152.2 \mathrm{~cm}$, and BMI of $39.1 \mathrm{~kg} / \mathrm{m}^{2}$. He showed no sign of diabetic retinopathy but he had microalbuminuria (13.6 mg/gCr). Despite having been treated with glibenclamide $(1.25 \mathrm{mg} /$ day $)$, metformin (1000 mg/day), and pioglitazone (15 mg/day), his HbA1c was $11.2 \%$ ( $99 \mathrm{mmol} / \mathrm{mol})$. He has never had a neuroleptic drug prescribed. After written informed consent was obtained from him and his parents, genetic testing was performed. Abnormal DNA methylation was detected by pyrosequencing in the DMR (differentially methylated region) upstream of the SNRPN (small nuclear ribonucleoprotein polypeptide N) gene. Single nucleotide polymorphism (SNP) array analysis was then performed using the SurePrint G3 ISCA CGH + SNP Microarray Kit (catalog number G4890A, Agilent Technologies); however copy number variants were not found. Finally, microsatellite analysis for chromosome 15 was performed, revealing that abnormal DNA methylation of the patient is due to uniparental (maternal) disomy (UPD) (Supplemental Fig. S1). Pharmacological treatment with metformin $(2.25 \mathrm{~g} /$ day $), \mathrm{NPH}$ insulin at bedtime of $28 \mathrm{U}$ to lower fasting glucose level, and miglitol $(150 \mathrm{mg} /$ day) were then administered in addition to a diet of $1600 \mathrm{kcal} /$ day with exercise therapy. Afterward, he lost $12 \mathrm{~kg}$ of fat rather than muscle in 7 months but his HbA1c level deteriorated to $12.2 \%(110 \mathrm{mmol} / \mathrm{mol})$. The fat mass and free fat mass were measured by bioimpedance analysis using Body Composition Analyzer MC-190 (standing-posture 8-electrode multi-frequency bioelectrical impedance analysis) (Tanita Corp., Tokyo, Japan). We then replaced NPH insulin with exenatide $(20 \mu \mathrm{g} /$ day) in expectation of a reduction of the increased appetite. However, his HbA1c fluctuated despite continuing to lose $15 \mathrm{~kg}$ body weight comprising $6 \mathrm{~kg}$ loss of fat and $9 \mathrm{~kg}$ loss of muscle. The massive loss of muscle mass 
during this period might be due to sustained hyperglycemia with relatively insufficient insulin. At the age of 33, his body weight reached $62.5 \mathrm{~kg}$. Apprehensive of further loss of muscle mass, basal insulin of insulin glargine ( $6 \mathrm{U} /$ day) and lixisenatide were administered in place of exenatide. Lixisenatide was anticipated to have improved usability with insulin glargine, both of which are once-daily injected drugs. After initiation of insulin glargine, his body weight was stabilized but his HbA1c did not improve. At the age of 35 , tofogliflozin ( $20 \mathrm{mg}$ /day) was added and lixisenatide was switched to dulaglutide ( $0.75 \mathrm{mg} /$ week). Just after the initiation of tofogliflozin, his HbA1c began to decrease. After 9 months, his body weight was maintained at $62.8 \mathrm{~kg}$ and $\mathrm{HbA1c}$ improved to
$8.5 \%(69 \mathrm{mmol} / \mathrm{mol})$ with a total ketone body level of $781 \mu \mathrm{M}$, which is within the range of physiological ketosis (Fig. 1). His C-peptide induced by glucagon did not differ significantly after the use of tofogliflozin (Supplementary Table S1). He then received an abdominal computer tomography (CT) scan. Compared with the findings at 28 years of age, his subcutaneous fat area (SFA) was decreased from 263.6 to $116.0 \mathrm{~cm}^{2}$ but his visceral fat area was hardly changed (Fig. 1). Abdominal CT images at 28 years of age showed severe fatty infiltration of the whole pancreas, but this disappeared in the CT images performed at 35 years of age. The mean Hounsfield unit (HU) of the regions of interest (ROIs) with areas of $1.0 \mathrm{~cm}^{2}$ was measured at pancreatic head $(P)$ and spleen $(S)$ on

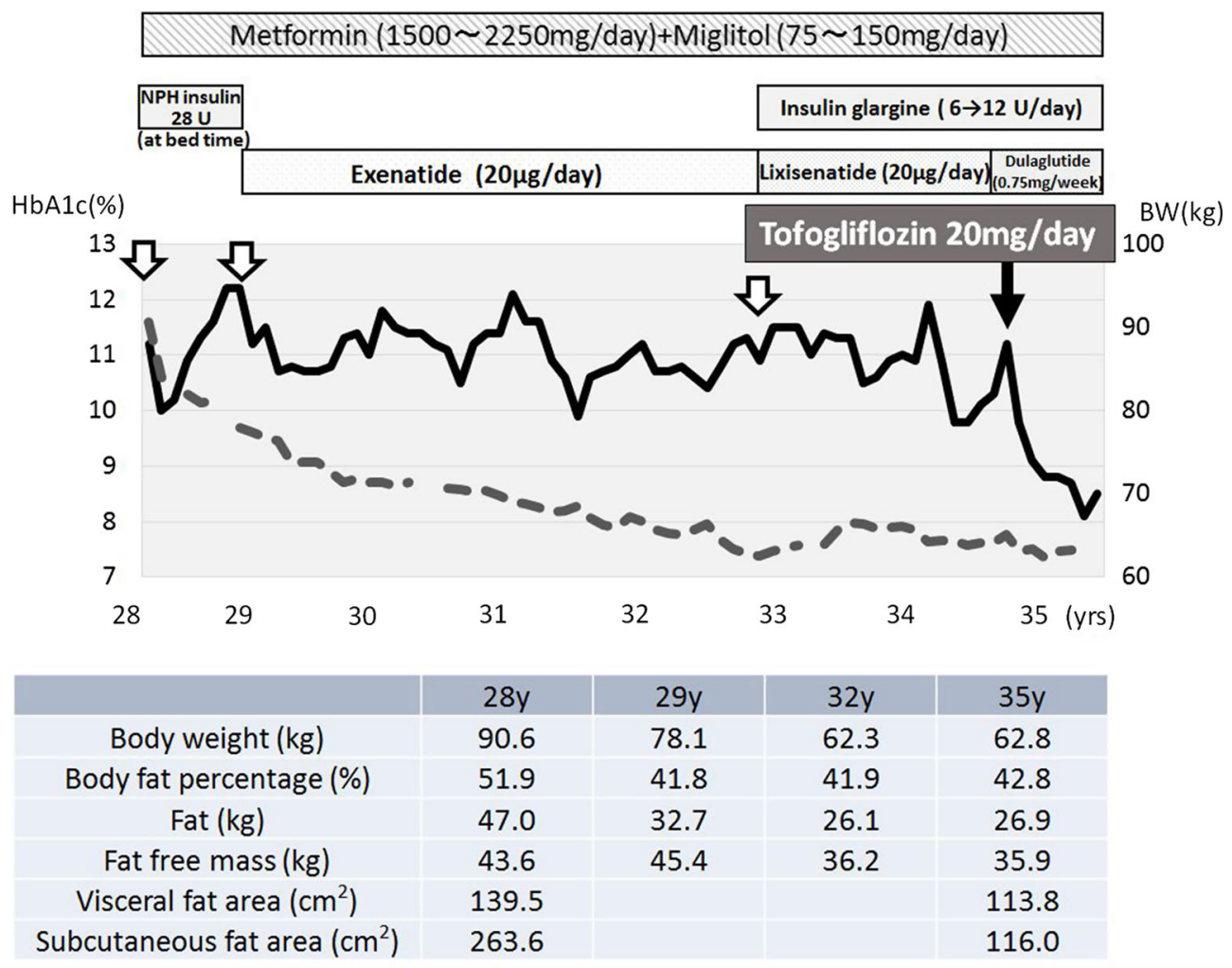

Fig. 1 Course of treatment graph showing the corresponding effects on weight and HbAlc. The duration of pharmacological agents is represented by the length of each box relative to the time period on the $\mathrm{x}$-axis. Changes in HbAlc are shown by the black line plotted against the $\mathrm{y}$-axis (left) and changes in weight are shown by the gray dotted line plotted against the y-axis (right). The black downward arrow indicates the time when tofogliflozin was begun. The white open arrows indicate hospitalization for glycemic control. Periodical change of body composition measurement was also shown in the lower panel 


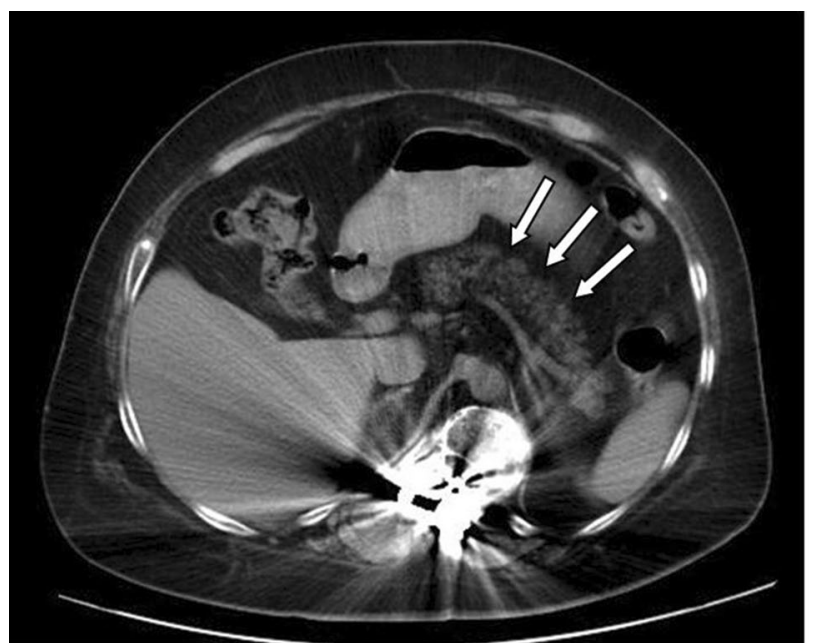

(A)

Fig. 2 Abdominal CT findings at 28 years (a) and 35 years (b) of age. a Abdominal CT imaging showing the presence of diffuse fat infiltration from the head to tail

CT images avoiding the metallic artifact from artificial rods for spinal fusion. Splenic attenuation was used for normalization of pancreatic attenuation. At 28 and 35 years of age, $P$ was -41.9 and $-2.6 \mathrm{HU}$, and $S$ was 48.9 and 60.0 HU, respectively. Accordingly, $P / S$ value improved drastically from -0.857 to -0.043 . Lipid deposition in the pancreas was extremely improved, while that in the liver did not differ significantly (Fig. 2). We continue to follow this patient at present and the effect has lasted at least 1 year. All procedures performed in the study were in accordance with the ethical standards of the institutional and/or national research committee and with the 1964 Helsinki declaration and its later amendments or comparable ethical standards. Written informed consent was obtained from all individual participants included in the study.

\section{DISCUSSION}

In the last two decades, the care of child and adult PWS has improved and the mortality rate seems to be declining [1]. The average age of patients with PWS is increasing, as is the number of patients with adult PWS being treated by physicians. However, information regarding the health problems of adults with PWS is still

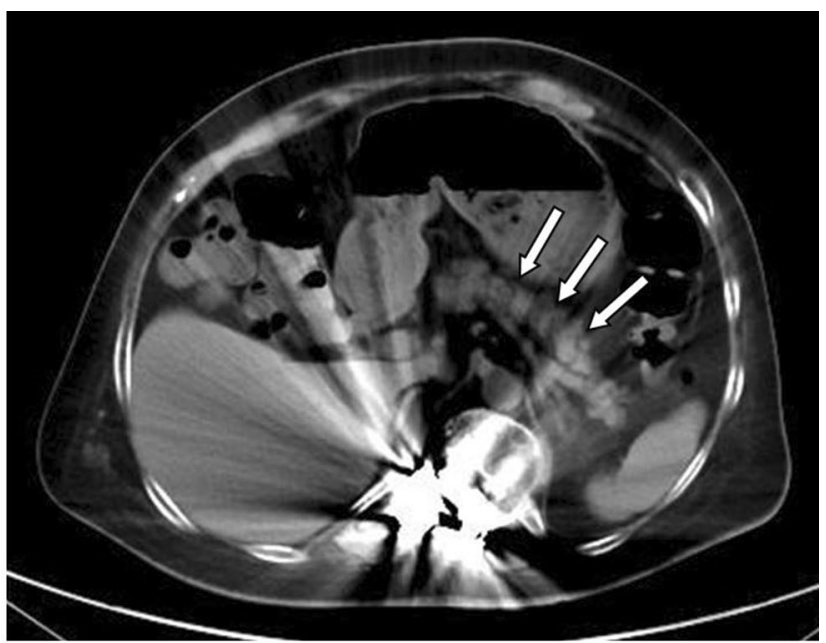

(B)

of the pancreas. $\mathbf{b}$ Relative to a, pancreatic normal tissue can be distinguished from the surrounding fat. The subcutaneous fat area has obviously declined

deficient. Most physical problems related to PWS are secondary to obesity, in which diabetes is well known to negatively influence the quality of life and life-span. Although it is uncommon for prepubertal children with PWS to develop overt diabetes, the prevalence of T2D in adults with PWS $(7-20 \%)$ is reported to be higher than that in the general population [2]. Moreover, Tsuchiya et al. reported that the onset of DM in patients with PWS in Japan is early, between 10 and 15 years of age, and that the overall frequency of DM is $26.2 \%$ [3].

Generally, diabetes patients with PWS are obese because of hyperphagia, and weight control by dietary modification and medicine is required for glycemic control. Additionally, there are several recent reports showing the effectiveness of GLP-1RAs for diabetes treatment in PWS [4-6], but the HbA1c in the present case was not improved by GLP-1RAs despite a 28-kg BW reduction. However, immediately after the addition of tofogliflozin, an SGLT2 inhibitor, the patient's HbA1c decreased dramatically with only about an additional 3\% BW reduction.

Recently, the effectiveness of combined use of GLP-1RAs and SGLT2 inhibitors has been recognized [7]. These drugs have several complementary features involving their mechanism of action [8]. While the elimination of glucose through the urine caused by SGLT2 inhibitors 
may stimulate appetite, this effect may be offset by the appetite-suppressing effect of GLP-1RAs that mitigates the deleterious effects of any weight gain. These drugs may therefore improve glycemic control, body weight, and cardiovascular risk when they are administered together. Additionally, in this case, mitigation of muscle loss after administration of basal insulin due to the addition of SGLT2 inhibitors may represent a further advantage of using these drugs in combination.

We should also note the improvement in our case of lipid deposition in the pancreas confirmed by abdominal CT after the improvement of HbA1c. The normal pancreatic fat cutoff point of $6.2 \%$ by MRI and more excessive pancreatic fat deposition are recommended to be considered non-alcoholic fatty pancreas disease (NAFPD) [9]. Although we could not estimate fatty mass itself by CT without MRI, it was very likely that the whole pancreas was infiltrated by fat, leading to the designation of NAFPD for the case. The relationship between CT indexes and histologically measured pancreatic fat was assessed, revealing that pancreatic fat could be quantified by using unenhanced CT [10]. The clinical importance of non-alcoholic fatty pancreas disease (NAFPD) has increasingly become known recently. NAFPD has been shown to exert adverse effects on pancreatic beta cell function and insulin action by in vitro and animal studies [11]. Although both NAFLD and NAFPD are strongly associated with visceral adipose tissue (VAT), the visceral fat area in the present case was barely changed, unlike the SFA. The improvement of fatty pancreas might improve insulin resistance rather than insulin secretion since the insulin secretory capacity was not changed significantly. It is unknown whether this improvement of fatty pancreas was a cause or an effect of improved glycemic control in the present case. Nevertheless, this finding clearly supports the effectiveness of combining SGLT2 inhibitors with GLP-1RAs for treatment of patients with PWS and NAFPD.

PWS is associated with many behavioral and psychiatric problems such as anxiety, temper outbursts, stubbornness, mood-related disorders, and learning difficulties. For this reason, careful clinical management by diet therapy is often unsuccessful. Hayashi et al. reported a case of PWS who developed severe ketoacidosis after administration of an SGLT2 inhibitor during a low-carbohydrate diet [12]. However, the principal factor for severe ketoacidosis in that case would seem to be the low-carbohydrate diet rather than the SGLT2 inhibitor therapy. Although it is possible that the obsessive-compulsive symptoms of PWS in that case affected adherence to the low-carbohydrate diet, medical staff should be instructed not to administer a low-carbohydrate diet when SGLT2 inhibitors are used in PWS.

\section{CONCLUSION}

This is the first report to show the effectiveness of SGLT2 inhibitors as an add-on drug to GLP1RAs for glycemic control of a patient with PWS and NAFPD. Further studies with more cases are required for confirmation, but the combination of these two medications would seem to ameliorate the prognosis of adults with PWS and NAFPD by improving glycemic control via the complementary actions of GLP-1RAs and SGLT2 inhibitors.

\section{ACKNOWLEDGEMENTS}

The authors are very grateful to the patient for his kind contribution to this study. We also thank Dr. Masami Mizuno and Tetsuya Suwa for collecting the laboratory data.

Funding. No funding or sponsorship was received for this study or publication of this article.

Authorship. All named authors meet the international Committee of Medical Journal Editors (ICMJE) criteria for authorship for this article, take responsibility for the integrity of the work as a whole, and have given their approval for this version to be published.

Disclosures. Yukio Horikawa received honoraria for lectures from Astellas Pharma Inc. and Kowa Company, Ltd. Jun Takeda received 
honoraria for lectures from Astellas Pharma Inc., Sanofi K.K, Ono Pharmaceutical Co. Ltd., Novo Nordisk Pharma Ltd., Eli Lilly Japan K.K., Daiichi Sankyo Co., Ltd., Takeda Pharmaceutical Co., Ltd., MSD., Dainippon Sumitomo Pharma Co., Ltd., Mitsubishi Tanabe Pharma Corporation, Boehringer Ingelheim, Taisho Toyama Pharmaceutical Co. Ltd. and Kowa Company, Ltd., and scholarship grants from Boehringer Ingelheim, Sanofi K.K, Ono Pharmaceutical Co. Ltd., Novo Nordisk Pharma Ltd., Novartis Pharma K.K., Sanwa Kagaku Kenkyusho Co., Ltd., Astellas Pharma Inc., Takeda Pharmaceutical Co., Ltd., Daiichi Sankyo Co., Ltd., Mitsubishi Tanabe Pharma Corporation, Eli Lilly Japan K.K. Taisho Toyama Pharmaceutical Co. Ltd., MSD., Kowa Company, Ltd., and Kyowa Hakko Kirin Co. Ltd. Mayumi Enya, Makie Komagata, Ken-ichi Hashimoto, Masayo Kagami, and Maki Fukami have nothing to disclose.

Compliance with Ethics Guidelines. All procedures performed in the study were in accordance with the ethical standards of the institutional and/or national research committee and with the 1964 Helsinki declaration and its later amendments or comparable ethical standards. Written informed consent was obtained from all individual participants included in the study.

Open Access. This article is distributed under the terms of the Creative Commons Attribution-NonCommercial 4.0 International License (http://creativecommons.org/licenses/ by-nc/4.0/), which permits any noncommercial use, distribution, and reproduction in any medium, provided you give appropriate credit to the original author(s) and the source, provide a link to the Creative Commons license, and indicate if changes were made.

\section{REFERENCES}

1. Seetho IW, Jones G, Thomson GA, et al. Aging in people with Prader-Willi syndrome: mortality in the UK population cohort and morbidity in an older sample of adults. Psychol Med. 2015;45:615-21.

2. Butler JV, Whittington JE, Holland AJ, et al. Prevalence of, and risk factors for, physical ill-health in people with Prader-Willi syndrome: a populationbased study. Dev Med Child Neurol. 2002;44:248-55.

3. Tsuchiya T, Oto Y, Ayabe T, Obata K, Murakami N, Nagai T. Characterization of diabetes mellitus in Japanese Prader-Willi syndrome. Clin Pediatr Endocrinol. 2011;20:33-8.

4. Sze L, Purtell L, Jenkins A, et al. Effects of a single dose of exenatide on appetite, gut hormones, and glucose homeostasis in adults with Prader-Willi syndrome. J Clin Endocrinol Metab. 2011;96:E1314-9.

5. Seetho IW, Jones G, Thomson GA, et al. Treating diabetes mellitus in Prader-Willi syndrome with exenatide. Diabetes Res Clin Pract. 2011;92:e1-2.

6. Fintini D, Grugni G, Brufani C, Bocchini S, Cappa $\mathrm{M}$, Crinò A. Use of GLP-1 receptor agonists in Prader-Willi syndrome: report of six cases. Diabetes Care. 2014;37:e76-7.

7. Busch RS, Kane MP. Combination SGLT2 inhibitor and GLP-1 receptor agonist therapy: a complementary approach to the treatment of type 2 diabetes. Postgrad Med. 2017;129:686-97.

8. Nauck MA, Meier JJ. GLP-1 receptor agonists and SGLT2 inhibitors: a couple at last? Lancet Diabetes Endocrinol. 2016;4:963-4.

9. Singh RG, Yoon HD, Wu LM, et al. Ectopic fat accumulation in the pancreas and its clinical relevance: a systematic review, meta-analysis, and meta-regression. Metabolism. 2017;69:1-13.

10. Kim SY, Kim H, Cho JY, et al. Quantitative assessment of pancreatic fat by using unenhanced CT: pathologic correlation and clinical implications. Radiology. 2014;271:104-12.

11. Yu TY, Wang CY. Impact of non-alcoholic fatty pancreas disease on glucose metabolism. J Diabetes Investig. 2017. https://doi.org/10.1111/jdi.12665.

12. Hayami $\mathrm{T}$, Kato $\mathrm{Y}$, Kamiya $\mathrm{H}$, et al. Case of ketoacidosis by a sodium-glucose cotransporter-2 inhibitor in a diabetic patient with a low-carbohydrate diet. J Diabetes Investig. 2015;6:587-90. 